\title{
Bacterial microbiota associated with Rhipicephalus sanguineus (s.l.) ticks from France, Senegal and Arizona
}

\author{
Magalie René-Martellet ${ }^{1,2 \dagger}$, Guillaume Minard ${ }^{3,4+}$, Raphael Massot ${ }^{1}$, Van Tran Van ${ }^{3}$, Claire Valiente Moro ${ }^{3}$, \\ Luc Chabanne $e^{1,2}$ and Patrick Mavingui $i^{3,5^{*}}$
}

\begin{abstract}
Background: Ticks of the group Rhipicephalus sanguineus (sensu lato) are distributed worldwide and are major pathogen vectors of both dogs and humans. Previous phylogenetic reconstructions have suggested the existence of two main lineages within this group, "Tropical" and "Temperate". Symbiotic interactions contribute to vector development, survival, reproduction and competence. The diversity of microbial communities associated with different populations of $R$. sanguineus (s.l.) remains poorly characterized, however, this knowledge will aid in future studies of hosts-microbiotapathogen interactions. To gain insight into the bacterial communities associated with $R$. sanguineus (s.l.) ticks, 40 specimens from France, Senegal and Arizona were analyzed by high-throughput 165 amplicon sequencing. All tick specimens were taxonomically classified using the mitochondrial 125 rDNA gene, which provides sufficient phylogenetic resolution to discriminate different lineages of $R$. sanguineus.
\end{abstract}

Results: Rhipicephalus sanguineus (s.l.) samples from Senegal belonged to the "Tropical" lineage, samples from France belonged to the "Temperate" lineage, whereas both lineages were identified in samples from Arizona. Regardless of origin, each bacterial microbiota was dominated by three genera: Coxiella, Rickettsia and Bacillus. Rickettsia and Coxiella were the two main genera found in females whereas males had a higher proportion of Bacillus. Significant differences of relative abundances were evidenced between specimens from different geographical origins.

Conclusions: This study highlights differences in the microbiota composition within R. sanguineus (s.l.) specimens from different genotypes, genders and geographical origins. This knowledge will help in future studies of the symbiotic interactions, biology and vector competence of the R. sanguineus (s.l.) complex.

Keywords: Rhipicephalus sanguineus, Symbionts, Coxiella, Rickettsia, Bacillus

\section{Background}

Infectious diseases are the second most common cause of death worldwide, behind cardiovascular diseases. Many arthropod vectors are known to transmit infection of "vector-borne diseases". The transmission cycles of such diseases rely on complex pathosystems in which vectors, hosts and microorganisms (from pathogens to mutualists)

\footnotetext{
* Correspondence: patrick.mavingui@cnrs.fr

${ }^{\dagger}$ Equal contributors

${ }^{3}$ Université de Lyon, Lyon, France Université Lyon 1, Villeurbanne, France;

CNRS, UMR 5557, Ecologie Microbienne, Villeurbanne, France; INRA,

UMR1418, Villeurbanne, France

${ }^{5}$ Université de La Réunion, CNRS UMR 9192, INSERM U1187, IRD UMR 249,

Unité Mixte Processus Infectieux en Milieu Insulaire Tropical (PIMIT),

Plateforme de Recherche CYROI, Saint-Denis, La Réunion, France

Full list of author information is available at the end of the article
}

interact within changing ecosystems. This complexity, and the diversity of players/partners involved in pathosystems, can make vector control difficult in the field. It has been demonstrated that some microbe-microbe interactions that occur within vectors, in particular mosquitoes, can interfere with life-history traits, including vector competence [1-6]. Consequently, increasing numbers of studies of vector-borne pathogen transmission and control now adopt integrative approaches that take all interacting players of each pathosystem into account.

Globally, ticks are considered as the second most important disease vectors after mosquitoes [7]. But in Europe, ticks are considered the most common vector of both human and veterinary diseases to date [8]. The last 
decades have witnessed the emergence of new tick-borne diseases and changes in the geographical distribution of previously known tick-borne pathogens. This suggests that the emergence and re-emergence of several tick-borne pathogens is likely to be of significant socioeconomic burden in the future [8-10].

Rhipicephalus sanguineus was first described by Latreille in 1806 using specimens collected from Gallia [11]. It is one of the most prevalent ticks found on dogs in southern France [12] and specimens attributed to the species have been described nearly worldwide. The taxonomic status of $R$. sanguineus remains controversial and has been subject to numerous debates over the last decades [11, 13-18]. The main conclusions of these studies referred to the absence of a reliable pictorial key to morphologically identify $R$. sanguineus (Latreille, 1806), the existence of different populations within the morphotype $R$. sanguineus and the possible confusion with $R$. turanicus. Therefore, all the specimens harboring the original pictorial keys annotated in the first description were gathered under the taxon $R$. sanguineus (sensu lato) (s.l.). Within $R$. sanguineus (s.l.), two parapatric lineages were identified based on phylogeny of mitochondrial $16 S$ and $12 S$ rDNA genes: a temperate southern American/western European lineage and a tropical southern American/African lineage [13, 17, 19].

Rhipicephalus sanguineus (s.l.) is highly specialized for dogs and, thus, occasionally colonizes human habitats. It is considered as a significant vector due to its ability to replicate and transmit many bacterial and parasitic agents, including Ehrlichia canis, Mycoplasma haemocanis, Babesia vogeli, Hepatozoon canis to carnivores and Rickettsia conorii, the agent of the Mediterranean spotted fever, to humans $[18,20]$. Because of its vector role, as well as its ability to colonize human habitats, this species is a real threat to human and animal health [21].

Metazoans interact with a broad community of microorganisms [22]. Those interactions are called symbiosis and can result in positive, negative or neutral effects on both partners [23, 24]. Symbiont-based strategies have been proposed as a tool for vector control in mosquitoes [3] as well as in ticks [25]. Such approaches will gain in efficiency if fundamental knowledge is acquired on host-microbiota-pathogens interactions and dynamics. One of the first steps to apply such strategies is the description of the microbial communities that interact with populations of $R$. sanguineus (s.l.) in natura. To date, few studies have aimed at identifying bacterial communities within $R$. sanguineus (s.l.). Analysis of specimens from laboratory colonies [26] or collected from the same area have shown the dominance of the genera Coxiella and Rickettsia, as well as the detection of the intracellular bacteria Wolbachia spp. [27, 28]. In particular, a multicentric study conducted in southern France from 2010 to 2012 showed significant regional differences in the prevalence of $R$. sanguineus (s.l.) ticks infection by Babesia vogeli, a hemoprotozoan pathogen of dogs [12]. As no genetic difference (using $16 S$ and $12 S$ rDNA fragments) was shown between tick specimens collected in the sampled regions, the possibility that such differences in prevalence of infection could be associated with differences in microbiota within $R$. sanguineus (s.l.) specimens was suggested [12].

To get further insight into bacterial communities hosted by $R$. sanguineus (s.l.) ticks, specimens were collected in four areas of southern France, one area in Arizona and one area in Senegal. To overcome misinterpretations linked to the controversial taxonomic status, all $R$. sanguineus (s.l.) specimens used were genetically characterized by sequencing of a $400 \mathrm{bp}$ mitochondrial $12 \mathrm{~S}$ rRNA fragment. Then, high-throughput 165 rRNA amplicon sequencing was performed to describe the diversity and structure of bacterial communities interacting with $R$. sanguineus (s.l.) from different origins.

\section{Methods \\ Sampling}

The survey was conducted in four areas from southern France (Corsica, Drôme, Gard and Var), one location in Senegal (Dakar area) and one location in USA (San Carlos area, Arizona) (Table 1). All study sites were selected because of the high prevalence of $R$. sanguineus (s.l.) ticks recorded from previous surveys. Sampling in Senegal and Arizona was performed in order to compare their bacterial microbiota to that of the French populations, encompassing the two lineages known as "Temperate" and "Tropical". The four French sites also allowed exploration of possible differences in bacterial communities of ticks from different locations within the same country. Ticks were collected from either infested dogs or the environment by visual picking, flagging or $\mathrm{CO}_{2}$ trapping. Adults and nymphs were collected at each site. All ticks were stored in $70 \%$ ethanol until used. Collected ticks were sorted by developmental stage (nymphs, adult males and females) and were identified under light microscopy using pictorial keys [29, 30], allowing selection of $R$. sanguineus (s.l.). Then ticks were randomly selected within each group to be as representative for each stage and location as possible. Taking into account the complex taxonomic status of $R$. sanguineus (s.l.), and the possible misidentification with other species of the genus, all morphological identification was confirmed by amplification and sequencing of a $400 \mathrm{bp}$ fragment of mitochondrial $12 S$ rRNA gene. In the case of ticks collected from dogs, only the least bloodengorged ticks were kept (Additional file 1: Table S1).

\section{DNA extraction}

DNA was extracted as previously described [31] and the quality assessed by PCR amplification of a 400 bp 
Table 1 Information on the 40 R. sanguineus (s.l.) specimens used for analyses of bacterial diversity

\begin{tabular}{|c|c|c|c|c|}
\hline Sample & Location (coordinates) & Sex/Stage & $\begin{array}{l}\text { Collection } \\
\text { method }\end{array}$ & Haplotype \\
\hline AR-1 & \multirow{5}{*}{$\begin{array}{l}\text { San Carlos, Arizona } \\
\left(33^{\circ} 20^{\prime} \mathrm{N}, 110^{\circ} 27 \mathrm{~W}\right)\end{array}$} & $\mathrm{F}$ & \multirow{5}{*}{$\begin{array}{l}\text { From the } \\
\text { environment }^{a}\end{array}$} & 5 \\
\hline AR-2 & & $\mathrm{F}$ & & 5 \\
\hline AR-3 & & M & & 5 \\
\hline AR-4 & & M & & 6 \\
\hline AR-5 & & M & & 5 \\
\hline FR-CO1 & \multirow{5}{*}{$\begin{array}{l}\text { Bastia, Corsica, France } \\
\left(42^{\circ} 41^{\prime} \mathrm{N}, 9^{\circ} 27^{\prime} \mathrm{E}\right)\end{array}$} & M & \multirow[t]{5}{*}{ From dogs } & 1 \\
\hline FR-CO2 & & $\mathrm{F}$ & & 1 \\
\hline FR-CO3 & & M & & 1 \\
\hline FR-CO4 & & $\mathrm{N}$ & & 1 \\
\hline FR-CO7 & & $\mathrm{F}$ & & 1 \\
\hline FR-D1 & \multirow{7}{*}{$\begin{array}{l}\text { La Bégude de Mazenc, } \\
\text { Drôme, France } \\
\left(44^{\circ} 32^{\prime} \mathrm{N}, 4^{\circ} 56^{\prime} \mathrm{E}\right)\end{array}$} & $\mathrm{F}$ & \multirow[t]{7}{*}{ From dogs } & 1 \\
\hline FR-D2 & & $\mathrm{F}$ & & 1 \\
\hline FR-D3 & & $\mathrm{F}$ & & 1 \\
\hline FR-D4 & & $\mathrm{F}$ & & 1 \\
\hline FR-D5 & & $\mathrm{F}$ & & 1 \\
\hline FR-D6 & & $\mathrm{F}$ & & 1 \\
\hline FR-D7 & & M & & 1 \\
\hline FR-G1 & \multirow{8}{*}{$\begin{array}{l}\text { Sommières, Gard, France, } \\
\left(43^{\circ} 47^{\prime} \mathrm{N}, 4^{\circ} 05^{\prime} \mathrm{E}\right)\end{array}$} & $\mathrm{F}$ & \multirow[t]{6}{*}{ From dogs } & 1 \\
\hline FR-G2 & & $\mathrm{F}$ & & 1 \\
\hline FR-G3 & & $\mathrm{F}$ & & 1 \\
\hline FR-G4 & & $\mathrm{F}$ & & 3 \\
\hline FR-G6 & & $\mathrm{N}$ & & 1 \\
\hline FR-G7 & & $\mathrm{N}$ & & 1 \\
\hline FR-G13 & & $\mathrm{F}$ & \multirow{2}{*}{$\begin{array}{l}\text { From the } \\
\text { environment }^{b}\end{array}$} & 3 \\
\hline FR-G14 & & $\mathrm{F}$ & & 1 \\
\hline FR-G5 & \multirow{2}{*}{$\begin{array}{l}\text { Aigues-Vives, Gard, France } \\
\left(43^{\circ} 42^{\prime} \mathrm{N}, 4^{\circ} 13^{\prime} \mathrm{E}\right)\end{array}$} & $\mathrm{F}$ & From a dog & 1 \\
\hline FR-G11 & & $\mathrm{F}$ & $\begin{array}{l}\text { From the } \\
\text { environment }\end{array}$ & 3 \\
\hline FR-G9 & $\begin{array}{l}\text { Saint-Gilles, Gard, France } \\
\left(43^{\circ} 40^{\prime} \mathrm{N}, 4^{\circ} 26^{\prime} \mathrm{E}\right)\end{array}$ & M & From dogs & 1 \\
\hline FR-V4 & \multirow{5}{*}{$\begin{array}{l}\text { Toulon, Var, France } \\
\left(43^{\circ} 07^{\prime} \mathrm{N}, 5^{\circ} 55^{\prime} \mathrm{E}\right)\end{array}$} & $\mathrm{F}$ & \multirow[t]{5}{*}{ From dogs } & 3 \\
\hline FR-V5 & & $\mathrm{F}$ & & 3 \\
\hline FR-V6 & & $\mathrm{F}$ & & 1 \\
\hline FR-V7 & & M & & 1 \\
\hline FR-V8 & & M & & 1 \\
\hline SEN-1 & \multirow{7}{*}{$\begin{array}{l}\text { Dakar, Senegal } \\
\left(14^{\circ} 43^{\prime} \mathrm{N}, 17^{\circ} 25 \mathrm{~W}\right)\end{array}$} & $\mathrm{F}$ & \multirow[t]{7}{*}{ From dogs ${ }^{d}$} & 7 \\
\hline SEN-3 & & $\mathrm{F}$ & & 7 \\
\hline SEN-4 & & $\mathrm{F}$ & & 8 \\
\hline SEN-5 & & $\mathrm{F}$ & & 7 \\
\hline SEN-6 & & M & & 6 \\
\hline SEN-7 & & M & & 8 \\
\hline SEN-8 & & $\mathrm{F}$ & & 7 \\
\hline
\end{tabular}

Abbreviations: $\mathrm{F}$, female; $\mathrm{M}$, male; $\mathrm{N}$, nymph

${ }^{a}$ Using $\mathrm{CO}_{2}$ traps in sub-urban private houses

bUsing the flagging method, in a rural location, along a small wooded river occasionally frequented by dogs

"Using the flagging method, along the river "Le Vidourle" in a park within this middle town of southern France

${ }^{d}$ Dogs from the same kennel fragment of mitochondrial $12 \mathrm{~S}$ rRNA gene using primers targeting ticks [19]. Quantification of total DNA was systematically performed after each DNA extraction using a spectrophotometer (SAFAS, Monaco, Principality of Monaco). For each series of extractions, a negative control corresponding to an extraction tube without a tick sample was performed in parallel.

\section{Rhipicephalus sanguineus (s.l.) haplotyping}

Products of PCR amplification of the mitochondrial $12 S$ rRNA genes of all $R$. sanguineus (s.l.) ticks used in the study were sequenced by BIOFIDAL-DTAMB (FR BioEnvironment and Health, Lyon, France). Sequences obtained were manually corrected by visual analysis of the electropherogram, aligned and assembled by haplotypes using Bioedit v7.0.5.3 [32]. The consensus sequence of each $R$. sanguineus (s.l.) haplotype was then used as query sequences using BLAST against the NCBI nucleotide database. The $400 \mathrm{bp}$ sequences of $R$. sanguineus (s.l.) ticks were then aligned with sequences of $R$. sanguineus (s.l.) from different parts of the world and a phylogenetic tree was built using the Maximum Likelihood method. The nucleotide evolution model of Hasegawa, Kishino and Yano was selected based on the Akaike Information Criterion corrected following the previously described method [33]. The mitochondrial $12 S$ rDNA sequences from ticks of $R$. sanguineus (s.l.) used in the study were deposited in the GenBank database under the accession numbers KU255848KU255856.

\section{Bacterial 16S rDNA analysis}

Amplification of V5-V6 rrs hypervariable regions was performed in triplicates as previously described [34]. Briefly, $30 \mathrm{ng}$ of DNA were amplified with $200 \mathrm{nM}$ of each primer, $1.75 \mathrm{U}$ of Expand High Fidelity Enzyme Mix (Roche, Basel, Switzerland), 1× Expand High Fidelity Buffer (Roche, Basel, Switzerland), $0.06 \mathrm{mg} / \mathrm{ml}$ of T4 gene 32 protein (New England Biolabs, Evry, France), $0.06 \mathrm{mg} / \mathrm{ml}$ of bovine serum albumin (New England Biolabs, Evry, France), and $40 \mu \mathrm{M}$ of dNTP. The 784F (5' -AGG ATT AGA TAC CCT GGT A-3') and 1061R (5'-CRR CAC GAG CTG ACG AC-3') primers used also contained an 8 bp multiplex barcode and Illumina adapters. Amplifications were performed on Biorad C1000 thermal cycler (Biorad, CA, USA), with 5 min at $95{ }^{\circ} \mathrm{C}, 35$ cycles with $40 \mathrm{~s}$ of denaturation at $95{ }^{\circ} \mathrm{C}, 1 \mathrm{~min}$ of hybridization at $54.2{ }^{\circ} \mathrm{C}, 30 \mathrm{~s}$ of extension at $72{ }^{\circ} \mathrm{C}$ and a final extension step of $7 \mathrm{~min}$ at $72{ }^{\circ} \mathrm{C}$. Amplicons were purified with Agencourt AMPure XP PCR Purification kit (Beckman Coulter, Villepinte, France) and quantified using the Quant-iT Picogreen dsDNA Assay Kit (Life Technologies, NY, USA). Then an equimolar mix of each amplicon was prepared for 
sequencing. A negative control (pool of 3 amplifications of a blank extraction) was sequenced with the library pool. Sequencing of libraries was performed on the Illumina Miseq Platform $(2 \times 250$-bp paredend reads) by ProfileXpert - ViroScan 3D (Lyon, France). Analysis of the V5-V6 rrs sequences was performed with Mothur v.1.33.3 pipeline [35]. Briefly, sequences were trimmed based on (i) index presence with less than two errors on primers; (ii) size comprised between $250 \mathrm{bp}$ and $350 \mathrm{bp}$; and (iii) no ambiguous sequences and less than 9 homopolymers. Sequences were then aligned on Silva v.119 and chimeras were removed with UCHIME implemented in Mothur [36]. A total of $8,972,702$ sequences of good quality were available for the analysis. According to neighbor-joining method, similar sequences were then clustered as a unique OTU if they harbored less than 3\% divergence. The taxonomy assignments of OTUs were performed with Naive Bayes Classifier using Silva database v.119. Contaminants were removed from the analysis using a homemade script that suppresses OTUs if their relative abundance (proportion of reads) in a given sample was not at least $10 \times$ higher than in the negative control. For all the analyses, an even number of sequences were used. Richness (sobs, chao1), $\alpha$-diversity $\left(1 / \lambda, H^{\prime}\right)$ and $\beta$ diversity (Bray-Curtis) indices were calculated (Additional file 1: Table S2). Non-parametric analysis of microbiota homogeneity (Homogeneity of Variance, HOMOVA) and differentiation (Analysis of Molecular Variance, AMOVA) among samples were performed with MOTHUR pipeline, following the method previously described [37]. The HOMOVA analysis compares the homogeneity of the communities within the groups and the AMOVA compares the differences of the microbial communities among the groups. Fastq files were deposited on the European Nucleotide Archive under the project accession number (PRJEB21785).

\section{Results}

Haplotyping of $R$. sanguineus (s.l.) ticks and samples selection

Forty-nine $R$. sanguineus (s.l.) ticks were first selected according to morphological criteria and named: AR-1-AR-5; FR-CO1-FR-CO7; FR-D1-FR-D7; FR-G1FR-G14; FR-V1-FR-V8; SEN-1-SEN-8, according to their geographical origin (AR: Arizona; FR-CO: FranceCorsica; FR-D: France-Drôme; FR-G: France-Gard; FR-V: France-Var; SEN: Senegal). Among these, 49 individuals, analyses of the $400 \mathrm{bp}$ segment of mitochondrial $12 \mathrm{~S}$ rRNA gene distinguished 8 different haplotypes (haplotypes 1-8). After BLAST analysis, ticks of haplotypes 1 (KU255848), 2 (KU255849), 3 (KU255850), 4 (KU 255851) and 5 (KU255852), all collected in France or Arizona, showed $99-100 \%$ homology with $R$. sanguineus (s.l.) ticks sequences from western Europe, USA and southern South America. Haplotypes 6 (KU255853 and KU255854), 7 (KU255855) and 8 (KU255856), were associated with ticks collected in Senegal, except one sample (AR-4) that was collected in Arizona. Those sequences showed 98-99\% of homology with $R$. sanguineus (s.l.) ticks sequences from Brazil (AY559842) and Asia (JX416325, DQ003001 and AY987377). The proportion of nucleotide identity between the $8 R$. sanguineus (s.l.) haplotype sequences identified in the study is presented in Table 2 .

Phylogenetic analysis (Fig. 1) confirmed the existence of two monophyletic clusters. The first cluster, labeled as the "Tropical" lineage, included $R$. sanguineus (s.l.) specimens from Asia and the northern part of South-America (Brazil). The second cluster, labelled as the "Temperate" lineage, included sequences from Europe, Egypt, USA and southern South America (Argentina, Uruguay). All $R$. sanguineus (s.l.) sequences of the study originating from Senegal clustered in the "Tropical" lineage, all $R$. sanguineus (s.l.) sequences from France clustered in the "Temperate" lineage whereas sequences of $R$. sanguineus (s.l.) from Arizona were distributed within both lineages. Interestingly,

Table 2 Sequence identity matrix between the eight $R$. sanguineus (s.l.) haplotypes detected in the study

\begin{tabular}{|c|c|c|c|c|c|c|c|c|}
\hline & haplotype $1^{\text {a }}$ & haplotype $2^{a}$ & haplotype $3^{\mathrm{a}}$ & haplotype $4^{a}$ & haplotype $5^{a}$ & haplotype $6^{\mathrm{b}}$ & haplotype $7^{\mathrm{b}}$ & haplotype $8^{\mathrm{b}}$ \\
\hline haplotype 1 & - & & & & & & & \\
\hline haplotype 2 & 0.982 & - & & & & & & \\
\hline haplotype 3 & 0.997 & 0.979 & - & & & & & \\
\hline haplotype 4 & 0.984 & 0.997 & 0.982 & - & & & & \\
\hline haplotype 5 & 0.994 & 0.977 & 0.997 & 0.979 & - & & & \\
\hline haplotype 6 & 0.745 & 0.742 & 0.745 & 0.745 & 0.742 & - & & \\
\hline haplotype 7 & 0.747 & 0.745 & 0.747 & 0.747 & 0.745 & 0.997 & - & \\
\hline haplotype 8 & 0.750 & 0.747 & 0.75 & 0.75 & 0.747 & 0.995 & 0.997 & - \\
\hline
\end{tabular}

${ }^{a} R$. sanguineus (s.l.) haplotypes of the "Temperate" lineage

${ }^{\mathrm{b}} R$. sanguineus (s.l.) haplotypes of the "Tropical" lineage 


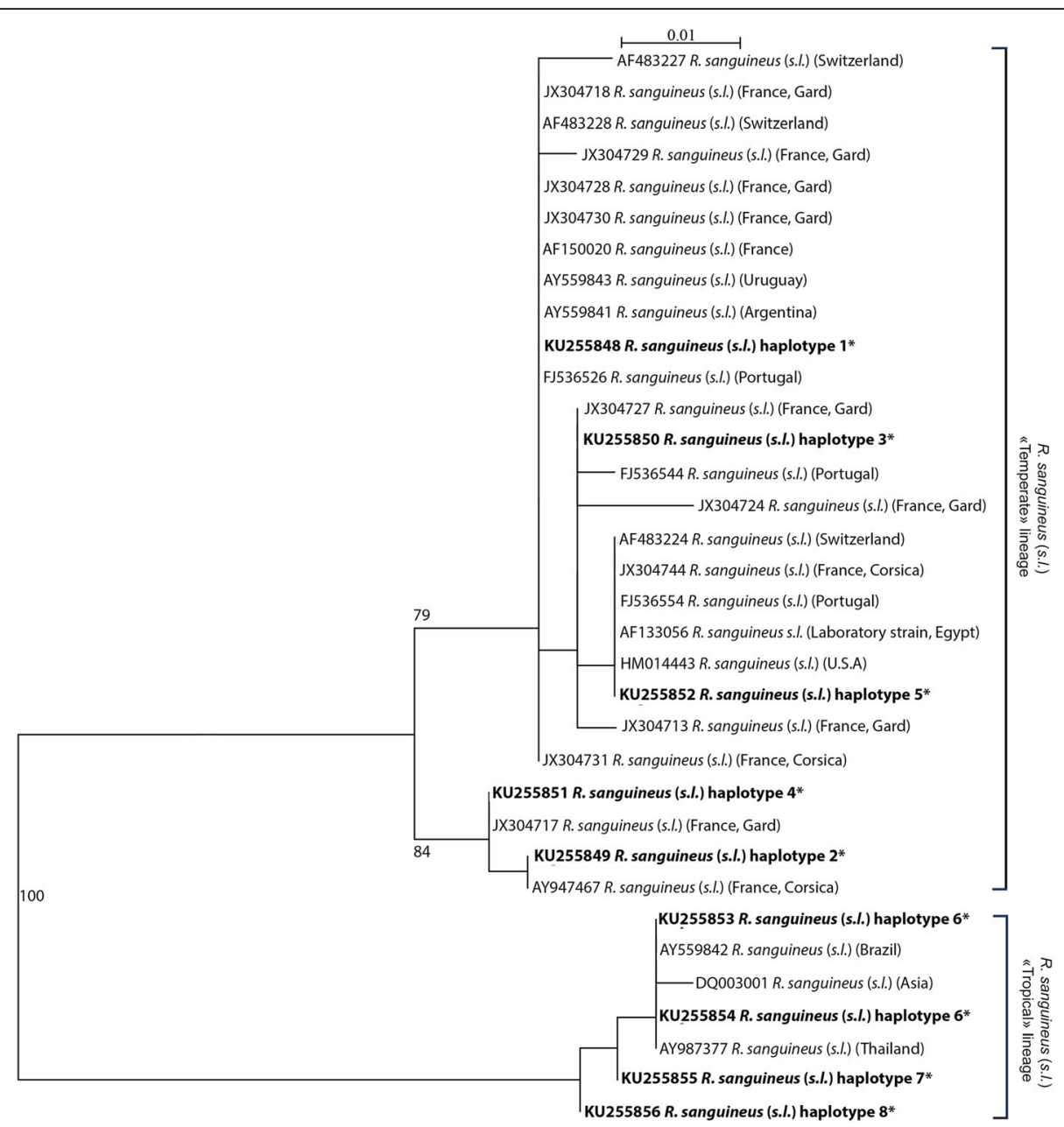

Fig. 1 Phylogenetic analysis of $R$. sanguineus (s.l.) ticks based on mitochondrial $12 S$ rRNA gene. Sequences (400 bp) of $R$. sanguineus (s.l.) ticks from France, Senegal and Arizona were assembled by haplotypes and compared to sequences of $R$. sanguineus (s.l.) from different parts of the world. Identification and GenBank accession numbers are indicated for each sample. Countries or regions where individuals were isolated are also given in brackets. The Maximum Likelihood phylogenetic tree was constructed using the Hasegawa, Kishino and Yano method with bootstrap analysis of 1000 pseudoreplicates. Numbers on branches indicate support for each clade $\geq 75 \%$. Subsequent analyses using Kimura's two-parameter (K2P) distance and parsimony methods in the same conditions confirmed the topology of the tree (not shown)

within the "Temperate" cluster, several specimens of $R$. sanguineus (s.l.) from France, including specimens of the study assigned to haplotypes 2 and 4, formed a monophyletic sub-group supported by a bootstrap value of $84 \%$.

Finally, among the 49 samples initially selected, two samples assigned to haplotypes 2 and 4 (FR-CO6 and FR-G8) were excluded from the study because of their genetic divergence within the "Temperate" lineage. Among the remaining ticks, five were discarded for insufficient amplification of the V5-V6 rrs hypervariable regions (samples FR-V1, FR-V2, FR-V3, FR-G10 and FR-G12) and 2 were excluded due to insufficient read number after Miseq sequencing (samples SEN-2 and FR-CO5). A total of 40 samples were kept for subsequent analyses of bacterial diversity. Information on the 40 analyzed samples is given in Table 1.

\section{Bacterial diversity}

The number of sequences obtained was between $85,388-386,478$ per sample. This depth was sufficient to reach reliable diversity estimates (Coverage > 0.999) (Additional file 1: Table S2). The bacterial estimated richness of $R$. sanguineus (s.l.) was comprised of between 34 and 562 OTUs per individual $($ Chaol $=243 \pm 132)$ (Additional file 1: Table S2). The bacterial microbiota of R. sanguineus (s.l.) was dominated by 3 genera (Fig. 2), namely Coxiella (phylum Proteobacteria, class Gammaproteobacteria), Rickettsia (phylum Proteobacteria, class Alphaproteobacteria) and Bacillus (phylum Firmicutes, class Bacilli). Both Coxiella and Rickettsia genera represented up to $99 \%$ of the total $R$. sanguineus (s.l.) microbiota. However, some samples had significantly higher proportions of Bacillus (up to 75\%). 


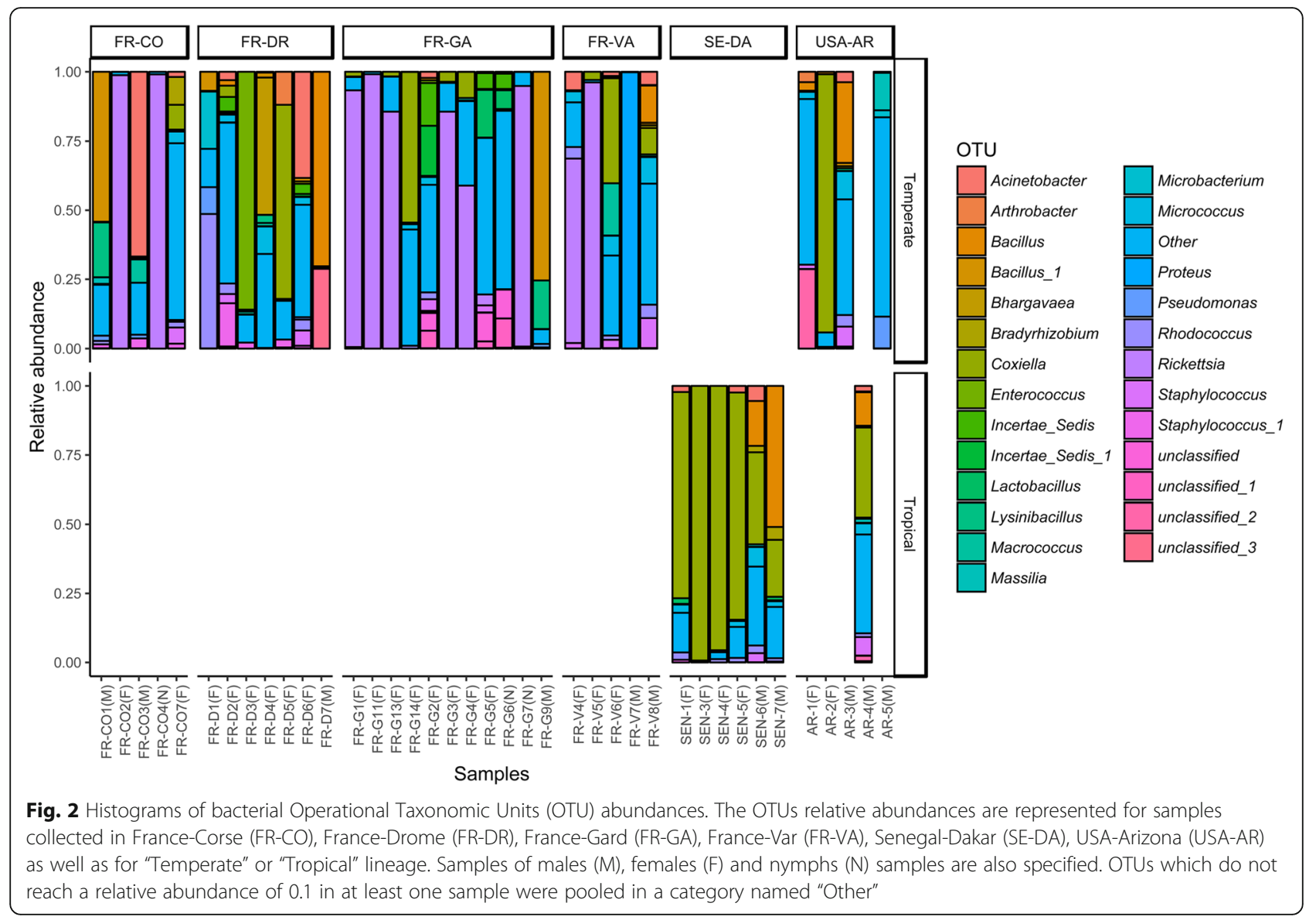

The effect of sample categories (sex/stage), origin, habitat and their interactions on the $\beta$-diversity was estimated by a permutational multivariate analysis of variance (adonis ANOVA test) with 999 permutations. Those three factors were correlated with $48 \%$ of the overall variability. Only the sample categories, namely males, females, nymph (adonis permutational ANOVA: $\left.F_{(2,28)}=2.29, P=0.013\right)$ and the origin (adonis permutational ANOVA: $F_{(2,28)}=2.02$, $P=0.002$ ) showed a difference in the microbiota similarity. Any differences in heterogeneity were evidenced between sample categories. The females harbored a microbiota dominated by both genera, Rickettsia and Coxiella, whereas the males were mainly associated with a higher proportion of Bacillus (Welsh corrected t-test: $t=-2.4, d f=10, P=0.03$ ) (Fig. 3a). In addition, adults harbored a higher proportion of Rhodococcus, Propionibacterium, Micrococcus and Bacillus, compared to nymphs (Fig. 3b). Significant differences in the microbiota homogeneity (higher within group similarity) and composition were observed between "Tropical" and "Temperate" tick lineages (HOMOVA: $B v=2.19, P<0.001$; AMOVA: $\left.F s_{(1,37)}=4.46, P=0.001\right)$ (Fig. $\left.3 \mathrm{~b}\right)$. However, the genetic divergence is confounded with the sampling site effect as the "Tropical" genotype was mainly associated with specimens collected from Senegal. Consequently, the differences between "Tropical" and Mediterranean populations reflected also the differences between Senegal and other locations. Indeed, Senegalese individuals harbored a more homogenous microbiota than those from France-Drôme and France-Gard (HOMOVA: $B v=1.75, P=0.002$ and $B v=1.19, P<0.001$, respectively). They also harbored a different microbiota from those collected in France-Corse, France-Gard and France-Var (AMOVA: $F s_{(1,9)}=4.64, P=0.002 ; F s_{(1,15)}=6.64, P<0.001$ and $F s_{(1,9)}=3.53, P=0.003$, respectively). Generally, a higher abundance of Coxiella was associated with Senegalese specimens, compared to those of the last three populations from France (Fig. 3c).

Within specimens collected from France, microbiota of female ticks collected in Gard was characterized by a high abundance of Rickettsia and Coxiella compared to ticks from the other three sites. However, the number of samples is not sufficient to generalize those observations with a statistical analysis. 

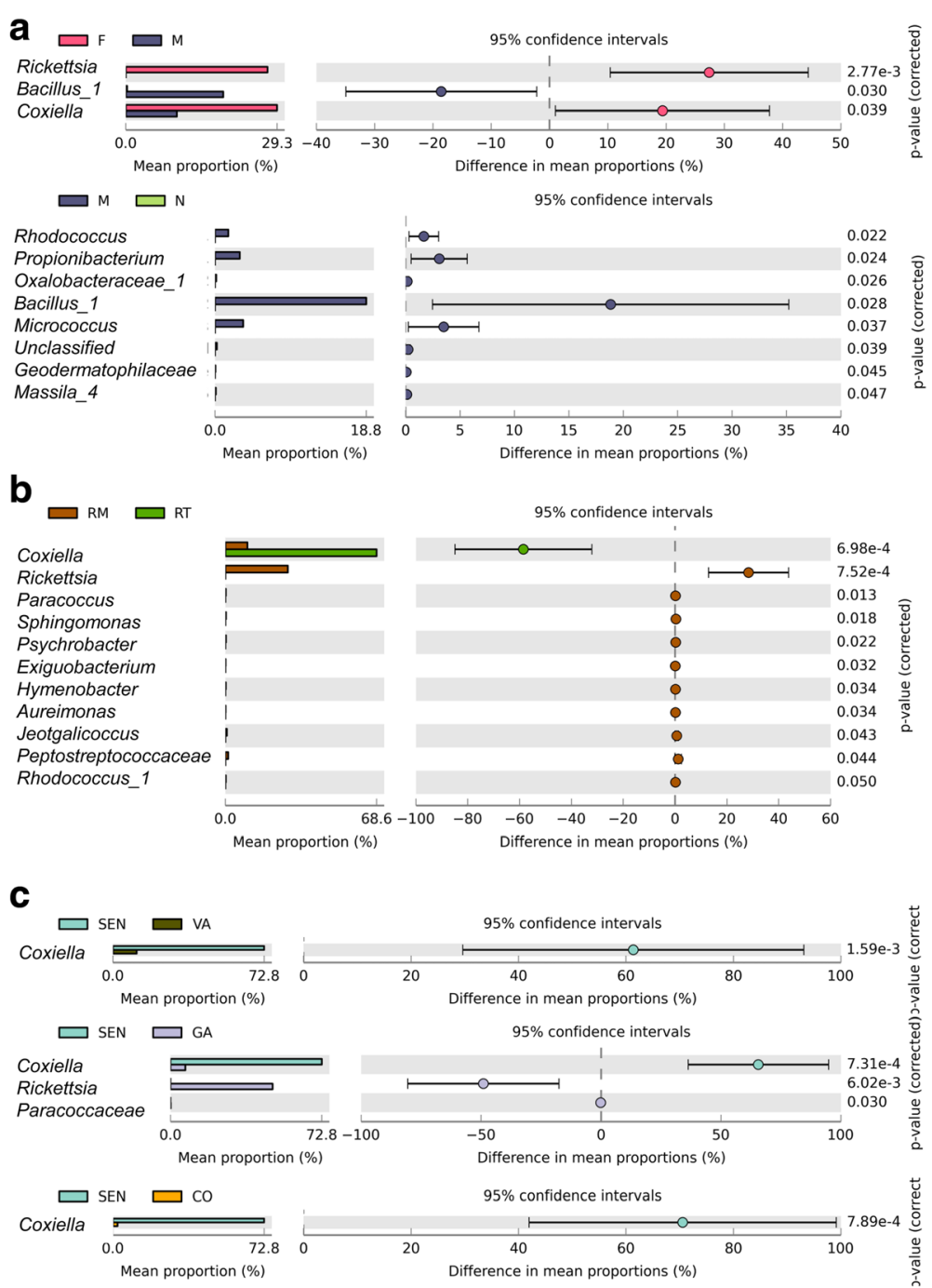

Fig. 3 Extended error bar plots of the most abundant OTUs associated with the covariates. Differences of abundances among OTUs were tested with a Welsh corrected $t$-test for R. sanguineus (s.l.) from: a Nymphs (N), females (F) and males (M); b "Tropical" lineage (RT) and "Temperate" lineage (RM); c Senegal (SEN); France-Var (VAR), France-Gard (GA) and France-Corsica (CO). The extended error bars represent the 95\% confidence interval of the fold change in relative abundance for an OTU between two modalities

\section{Discussion}

Few studies have aimed at identifying bacterial communities within ticks to date [25-28]. To provide new insight in bacterial communities hosted by $R$. sanguineus (s.l.) ticks, a survey of natural tick populations was conducted in 2014 in four locations at southern France, as well as in one site in Senegal and one site in Arizona. Taking into account the current controversial taxonomic status of $R$. sanguineus (s.l.) ticks, all specimens used in the study were genetically characterized by sequencing of a 400 bp mitochondrial $12 S$ rRNA fragment. As expected, all $R$. sanguineus (s.l.) sequences associated with Senegalese and French individuals were associated with the "Tropical" and "Temperate" lineages, respectively. In Arizona, ticks of both lineages were detected, which suggested that individuals from those two groups live in sympatry within this site. Two specimens were discarded from subsequent analyses because of their genetic divergence with the genotype of the most frequently encountered under "temperate" regions in the study. Analysis of bacterial communities within those particular specimens would result in difficulties in results interpretation.

High-throughput screening of variable V5-V6 bacterial $16 S$ rRNA gene was performed on all the forty selected $R$. sanguineus (s.l.) specimens. We found that the bacterial microbiota of $R$. sanguineus (s.l.) was dominated by three genera, namely Coxiella, Rickettsia and Bacillus representing up to 99\% (Coxiella and Rickettsia) and $75 \%$ (Bacillus) of the total relative abundance. Interestingly, females harbored a microbiota dominated by 
Rickettsia and Coxiella, whereas males harbored a higher proportion of Bacillus. Previous surveys on both genders have also shown that $R$. sanguineus (s.l.) ticks were mainly infected by the symbiotic/pathogenic bacterial genera Coxiella and Rickettsia [27, 28, 38]. Coxiella-like endosymbionts have already been shown to be mainly associated with females [27]. They can colonize Malpighian tubules, ovaries of females and can also be maternally transmitted [27, 28]. If no clear tissue tropism was detected for Rickettsia sp. in R. sanguineus (s.l.), maternal transmission of such symbionts has previously been observed [28, 39-41]. Lalzar et al. also suggested that a competition for the ovary colonization occurs between Coxiella sp. and Rickettsia sp. [28]. Our results support such hypothesis, since individuals infected by Rickettsia spp. or Coxiella spp. bacteria were mainly females and were found dominantly infected by either one of those symbionts, but never both at the same abundance.

Coxiella-like endosymbionts are widespread among the hard ticks, in which they present patterns of coevolution and genome reduction, which often occur in vertically-inherited endosymbionts [38, 42, 43]. Genome comparisons between Coxiella-like endosymbiont from the tick Amblyomma americanum and the $\mathrm{Q}$ fever ethiological agent Coxiella burnetii revealed an enrichment of genes involved in B vitamins and cofactors metabolism within the genome of the symbiotic strain [43]. The authors suggested that those bacterial functions are involved in a mutualistic interaction with the tick host by compensating nutritional deficiencies. Such a hypothesis is consistent with the observed decreases in ticks fecundity and life-history traits successive to the symbiont elimination [44]. The possible impact of Coxiella spp. and Rickettsia spp. on R. sanguineus (s.l.) survival, reproduction and vector competence needs to be explored further as it could open avenues for new control strategies.

Several species from the genus Bacillus were shown to colonize Arthropods. As an example, Bacillus thuringiensis, producing the Cry and Cyt toxins, has been broadly used for its biopesticide properties on Insects, whichhave shown interesting properties on Acarians [45, 46]. Bacillus spp. have previously been detected in several tick genera such as Ixodes, Amblyomma, Aponomma, Haemaphysalis and the species Rhipicephalus (Boophilus) microplus [47]. In the present study, Bacillus spp. represent $75 \%$ of the total relative abundance within $R$. sanguineus (s.l.) ticks and was mostly associated with males. The nature of the association between male $R$. sanguineus (s.l.) ticks and Bacillus spp. has not yet been characterized.

Finally, our results suggest variations of microbiota composition within $R$. sanguineus (s.l.) ticks related to origin. Similarly, Ixodes ticks originating from eastern USA presented a significant differentiation of their microbiota according to the distance among local populations [48]. In that study, variations in Rickettsia spp. and Enterobacteriaceae abundances were mainly responsible for those differentiations. In $R$. sanguineus (s.l.), site variations were related to a shift in the dominant taxa of Coxiella spp. to Rickettsia spp., and could be attributed to habitats or genetic changes associated with the ticks' populations belonging to "Temperate" or "Tropical" lineages. Interestingly, $R$. sanguineus (s.l.) ticks from Gard are associated with a highest relative abundance of Rickettsia compared to other French sampling sites. The Gard region has also been identified as a hotspot for the prevalence of the hemoprotozoan Babesia vogeli in dogs [12]. During the development of the Babesia protozoa in the tick the following events occur: (i) sexual reproduction in the lumen of the digestive tract then (ii) invasion of different organs, including Malpighian tubules and the ovaries $[49,50]$. Further investigations should be conducted to determine whether interactions between bacteria and the protozoa occur in ticks, and if they could interfere with its development or transmission as it has been previously shown with Plasmodium falciparum in mosquitoes [51].

Even though we have selected ticks that were not visually engorged, samples from some regions were collected on dogs and might have already ingested blood. Blood ingestion is responsible of transient changes in the microbial communities, and might have had an influence on our results [52]. More controlled studies should be conducted in order to tease apart those effects.

\section{Conclusions}

This study used a high-throughput sequencing approach to characterize the bacterial microbiota associated with $R$. sanguineus (s.l.) ticks obtained from different origins. Our results highlight differences in the structure of the microbiota, according to tick genotypes, geographical origin of specimens and stages (nymphs, male or female). Three dominant bacterial genera, namely Rickettsia, Coxiella and Bacillus, emerged from these analyzes with strong correlations with the samples category, geographical origin and lineage. The genus Coxiella is strongly associated with "Tropical" ticks from Africa whereas the genus Rickettsia is mainly found in "Temperate" ticks from the Gard region of France. This study is the first comprehensive overview of the structure of the microbiota, and its variation factors, within $R$. sanguineus (s.l.) ticks. These results provide a basis for future work on symbiotic interactions, biology and vector competence within the $R$. sanguineus (s.l.) complex. 


\section{Additional file}

Additional file 1: Table S1. Samples used for the $16 \mathrm{~s}$ rDNA high throughput sequencing. Table S2. Alpha diversity indices. (DOCX 23 kb)

\section{Abbreviations}

AMOVA: Analysis of molecular variance; ANOVA: Analysis of variance;

HOMOVA: Homogeneity of molecular variance; OTU: Operational taxonomic units; s.l:: sensu lato; s.s.: sensu stricto

\section{Acknowledgments}

We are grateful to Oleg Mediannikov (Institut de Recherche pour le Développement, UMR URMITE, Marseille, France) and Michael L. Levin (Centers for Disease Control and Prevention, Atlanta, USA) for sending specimens of $R$. sanguineus (s.l.) from Senegal and Arizona, respectively.

\section{Funding}

Financial support was provided by the CNRS-INEE through the Programme PEPS-Ecologie de la Santé ECOSAN 2014, project Ecopathotique by PM.

\section{Availability of data and materials}

All data generated or analyzed during this study are included in this published article and its Additional file. The sequences are submitted in the GenBank database under the accession numbers KU255848-KU255856 and in the European Nucleotide Archive under the project accession number PRJEB21785.

\section{Authors' contributions}

MR-M, CVM, LC and PM conceived the project and sampling design. MR-M collected the specimens and performed morphological, molecular identifications and phylogenetic reconstructions of ticks. MR-M, RM and VTV performed the molecular work. GM performed the bioinformatic analysis and statistical analysis. MR-M and GM wrote the first draft of the manuscript. All the authors contributed to the manuscript revision. All authors read and approved the final manuscript.

\section{Ethics approval and consent to participate}

Not applicable.

\section{Consent for publication}

Not applicable.

\section{Competing interests}

The authors declare that they have no competing interests.

\section{Publisher's Note}

Springer Nature remains neutral with regard to jurisdictional claims in published maps and institutional affiliations.

\section{Author details}

'VetAgro Sup campus vétérinaire de Lyon, Université de Lyon, Marcy l'Etoile, France. ${ }^{2}$ UMR 0346 EPIA, INRA-VetAgro Sup, Saint Genès Champanelle, Marcy I'Etoile, France. ${ }^{3}$ Université de Lyon, Lyon, France Université Lyon 1, Villeurbanne, France; CNRS, UMR 5557, Ecologie Microbienne, Villeurbanne, France; INRA, UMR1418, Villeurbanne, France. ${ }^{4}$ Metapopulation Research Centre, Department of Biosciences, University of Helsinki, Helsinki, Finland. 5Université de La Réunion, CNRS UMR 9192, INSERM U1187, IRD UMR 249, Unité Mixte Processus Infectieux en Milieu Insulaire Tropical (PIMIT)،

Plateforme de Recherche CYROI, Saint-Denis, La Réunion, France.

Received: 10 April 2017 Accepted: 29 August 2017

Published online: 07 September 2017

\section{References}

1. Bian G, Xu Y, Lu P, Xie Y, Xi Z. The endosymbiotic bacterium Wolbachia induces resistance to dengue virus in Aedes aegypti. PLoS Pathog. 2010;6: e1000833.

2. Brelsfoard $C L$, Séchan $Y$, Dobson SL. Interspecific hybridization yields strategy for South Pacific filariasis vector elimination. PLoS Negl Trop Dis. 2008;2:e129.
3. Moreira LA, Saig E, Turley AP, Ribeiro JMC, O'Neill SL, McGraw EA. Human probing behavior of Aedes aegypti when infected with a life-shortening strain of Wolbachia. PLoS Negl Trop Dis. 2009;3:e568.

4. Mousson L, Zouache K, Arias-Goeta C, Raquin V, Mavingui P, Failloux A-B. The native Wolbachia symbionts limit transmission of dengue virus in Aedes albopictus. PLoS Negl Trop Dis. 2012;6:e1989.

5. Minard G, Mavingui P, Moro CV. Diversity and function of bacterial microbiota in the mosquito holobiont. Parasit Vectors. 2013;6:146.

6. Kramer LD, Ciota AT. Dissecting vectorial capacity for mosquito-borne viruses. Curr Opin Virol. 2015;15:112-8.

7. de la Fuente J, Estrada-Pena A, Venzal JM, Kocan KM, Sonenshine DE. Overview: ticks as vectors of pathogens that cause disease in humans and animals. Front Biosci J Virtual Libr. 2008:13:6938-46.

8. Randolph SE. To what extent has climate change contributed to the recent epidemiology of tick-borne diseases? Vet Parasitol. 2010;167:92-4.

9. Beugnet F, Marié J-L. Emerging arthropod-borne diseases of companion animals in Europe. Vet Parasitol. 2009:163:298-305.

10. Dantas-Torres F, Chomel BB, Otranto D. Ticks and tick-borne diseases: a one health perspective. Trends Parasitol. 2012:28:437-46.

11. Nava S, Estrada-Peña A, Petney T, Beati L, Labruna MB, Szabó MPJ, et al. The taxonomic status of Rhipicephalus sanquineus (Latreille, 1806). Vet Parasitol. 2015;208:2-8.

12. René-Martellet M, Moro CV, Chêne J, Bourdoiseau G, Chabanne L, Mavingui P. Update on epidemiology of canine babesiosis in southern France. BMC Vet Res. 2015;11:223.

13. Hekimoğlu O, Sağlam IK, Özer N, Estrada-Peña A. New molecular data shed light on the global phylogeny and species limits of the Rhipicephalus sanguineus complex. Ticks Tick-Borne Dis. 2016;7:798-807.

14. Dantas-Torres F, Latrofa MS, Annoscia G, Giannelli A, Parisi A, Otranto D. Morphological and genetic diversity of Rhipicephalus sanguineus sensu lato from the new and old worlds. Parasit Vectors. 2013;6:213.

15. Dantas-Torres F, Otranto D. Further thoughts on the taxonomy and vector role of Rhipicephalus sanguineus group ticks. Vet Parasitol. 2015;208:9-13.

16. Latrofa MS, Dantas-Torres F, Annoscia G, Cantacessi C, Otranto D. Comparative analyses of mitochondrial and nuclear genetic markers for the molecular identification of Rhipicephalus spp. Infect Genet Evol. 2013;20:422-7.

17. Moraes-Filho J, Marcili A, Nieri-Bastos FA, Richtzenhain LJ, Labruna MB. Genetic analysis of ticks belonging to the Rhipicephalus sanguineus group in Latin America. Acta Trop. 2011;117:51-5.

18. Dantas-Torres F. The brown dog tick, Rhipicephalus sanguineus (Latreille, 1806) (Acari: Ixodidae): from taxonomy to control. Vet Parasitol. 2008;152:173-85.

19. Szabó MPJ, Mangold AJ, João CF, Bechara GH, Guglielmone AA. Biological and DNA evidence of two dissimilar populations of the Rhipicephalus sanguineus tick group (Acari: Ixodidae) in South America. Vet Parasitol. 2005;130:131-40.

20. Solano-Gallego L, Baneth G. Babesiosis in dogs and cats - expanding parasitological and clinical spectra. Vet Parasitol. 2011;181:48-60.

21. Day MJ. One health: the importance of companion animal vector-borne diseases. Parasit Vectors. 2011;4:49.

22. McFall-Ngai M, Hadfield MG, Bosch TCG, Carey HV, Domazet-Lošo T, Douglas $A E$, et al. Animals in a bacterial world, a new imperative for the life sciences. Proc Natl Acad Sci USA. 2013:110:3229-36.

23. Douglas AE. Symbiotic interactions. Oxford, New York: Oxford University Press; 1994

24. Douglas AE. The symbiotic habit. New Jersey: Princeton University Press; 2010.

25. Gall CA, Reif KE, Scoles GA, Mason KL, Mousel M, Noh SM, et al. The bacterial microbiome of Dermacentor andersoni ticks influences pathogen susceptibility. ISME J. 2016;10:1846-55.

26. Noda H, Munderloh UG, Kurtti TJ. Endosymbionts of ticks and their relationship to Wolbachia spp. and tick-borne pathogens of humans and animals. Appl Environ Microbiol. 1997:63:3926-32.

27. Lalzar I, Harrus S, Mumcuoglu KY, Gottlieb Y. Composition and seasonal variation of Rhipicephalus turanicus and Rhipicephalus sanguineus bacterial communities. Appl Environ Microbiol. 2012;78:4110-6.

28. Lalzar I, Friedmann Y, Gottlieb Y. Tissue tropism and vertical transmission of Coxiella in Rhipicephalus sanguineus and Rhipicephalus turanicus ticks. Environ Microbiol. 2014:16:3657-68.

29. Estrada-Peña A, Bouattour A, Camicas JL, Walker AR. Ticks of domestic animals in the Mediterranean region: a guide to identification of species. Zaragoza: University of Zaragoza; 2004. 
30. Pérez-Eid C. Les tiques. Identification, biologie, importance médicale et vétérinaire. Editions TEC\&DOC. Paris: Editions Médicales Internationales, Cachan; 2007.

31. René M, Chêne J, Beaufils JP, Valiente Moro C, Bourdoiseau G, Mavingui P, et al. First evidence and molecular characterization of Babesia vogeli in naturally infected dogs and Rhipicephalus sanguineus ticks in southern France. Vet Parasitol. 2012;187:399-407.

32. Hall TA. BioEdit: a user-friendly biological sequence alignment editor and analysis program for windows 95/98/NT. Nucl Acids Symp Ser. 1999;41:95-8.

33. Posada D. jModelTest: phylogenetic model averaging. Mol Biol Evol. 2008;25:1253-6.

34. Minard G, Tran F-H, Dubost A, Tran-Van V, Mavingui P, Moro CV. Pyrosequencing 16S rRNA genes of bacteria associated with wild tiger mosquito Aedes albopictus: a pilot study. Front Cell Infect Microbiol. 2014;4:59.

35. Schloss PD, Westcott SL, Ryabin T, Hall JR, Hartmann M, Hollister EB, et al. Introducing mothur: open-source, platform-independent, communitysupported software for describing and comparing microbial communities. Appl Environ Microbiol. 2009;75:7537-41.

36. Edgar RC, Haas BJ, Clemente JC, Quince C, Knight R. UCHIME improves sensitivity and speed of chimera detection. Bioinformatics. 2011;27:2194-200.

37. Schloss PD. Evaluating different approaches that test whether microbial communities have the same structure. ISME J. 2008;2:265-75.

38. Duron O, Noël V, McCoy KD, Bonazzi M, Sidi-Boumedine K, Morel O, et al. The recent evolution of a maternally-inherited endosymbiont of ticks led to the emergence of the Q Fever pathogen, Coxiella burnetii. PLoS Pathog. 2015;11:e1004892.

39. Socolovschi C, Bitam I, Raoult D, Parola P. Transmission of Rickettsia conorii conorii in naturally infected Rhipicephalus sanguineus. Clin Microbiol Infect. 2009;15:319-21.

40. Matsumoto K, Ogawa M, Brouqui P, Raoult D, Parola P. Transmission of Rickettsia massiliae in the tick, Rhipicephalus turanicus. Med Vet Entomol. 2005;19:263-70.

41. da Silva Costa LF, Nunes PH, Soares JF, Labruna MB, Camargo-Mathias MI. Distribution of Rickettsia rickettsii in ovary cells of Rhipicephalus sanguineus (Latreille, 1806) (Acari: Ixodidae). Parasit Vectors. 2011;4:222.

42. Machado-Ferreira E, Vizzoni VF, Balsemão-Pires E, Moerbeck L, Gazeta GS, Piesman J, et al. Coxiella symbionts are widespread into hard ticks. Parasitol Res. 2016;115:4691-9.

43. Smith TA, Driscoll T, Gillespie JJ, Raghavan R. A Coxiella-like endosymbiont is a potential vitamin source for the lone star tick. Genome Biol Evol. 2015;7:831-8.

44. Zhong J, Jasinskas A, Barbour AG. Antibiotic treatment of the tick vector Amblyomma americanum reduced reproductive fitness. PLoS One. 2007;2:e405.

45. Fernández-Ruvalcaba M, Peña-Chora G, Romo-Martínez A, HernándezVelázquez V, de la Parra AB, De La Rosa DP. Evaluation of Bacillus thuringiensis pathogenicity for a strain of the tick, Rhipicephalus microplus, resistant to chemical pesticides. J Insect Sci. 2010;10:186.

46. Dunstand-Guzmán E, Peña-Chora G, Hallal-Calleros C, Pérez-Martínez M, Hernández-Velazquez VM, Morales-Montor J, et al. Acaricidal effect and histological damage induced by Bacillus thuringiensis protein extracts on the mite Psoroptes cuniculi. Parasit Vectors. 2015;8:285.

47. Murrell A, Dobson SJ, Yang X, Lacey E, Barker SC. A survey of bacterial diversity in ticks, lice and fleas from Australia. Parasitol Res. 2003;89:326-34.

48. Treuren W, Ponnusamy L, Brinkerhoff RJ, Gonzalez A, Parobek CM, Juliano $J$ J, et al. Variation in the microbiota of Ixodes ticks with geography, species and sex. Appl Environ Microbiol. 2015;81:6200-9.

49. Chauvin A, Moreau E, Bonnet S, Plantard O, Malandrin L. Babesia and its hosts: adaptation to long-lasting interactions as a way to achieve efficient transmission. Vet Res. 2009;40:37.

50. Mehlhorn H, Schein E. The Piroplasms: life cycle and sexual stages. Adv Parasitol. 1984;23:37-103.

51. Dong Y, Manfredini F, Dimopoulos G. Implication of the mosquito midgut microbiota in the defense against malaria parasites. PLoS Pathog. 2009;5: e1000423.

52. Zhang X-C, Yang Z-N, Lu B, Ma X-F, Zhang C-X, Xu H-J. The composition and transmission of microbiome in hard tick, IXodes persulcatus, during blood meal. Ticks Tick-Borne Dis. 2014;5:864-70.

\section{Submit your next manuscript to BioMed Central and we will help you at every step:}

- We accept pre-submission inquiries

- Our selector tool helps you to find the most relevant journal

- We provide round the clock customer support

- Convenient online submission

- Thorough peer review

- Inclusion in PubMed and all major indexing services

- Maximum visibility for your research

Submit your manuscript at www.biomedcentral.com/submit
Biomed Central 